PROCEEDINGS OF THE

AMERICAN MATHEMATICAL SOCIETY

Volume 140, Number 2, February 2012, Pages 505-511

S 0002-9939(2011)10932-8

Article electronically published on June 22, 2011

\title{
GLOBAL SECTIONS OF STRUCTURE SHEAVES OF KEIGHER RINGS
}

\author{
DMITRY TRUSHIN \\ (Communicated by Bernd Ulrich) \\ This paper is dedicated to Jerald J. Kovacic
}

\begin{abstract}
Answering a question of J. Kovacic, we show that for any Keigher ring, its differential spectrum coincides with the differential spectrum of the ring of global sections of the structure sheaf. In particular, we obtain the answer for Ritt algebras, that is, differential rings containing the rational numbers.
\end{abstract}

\section{INTRODUCTION}

The study of differential schemes began in [7, 8, 9, 10, 11, and was continued in [4, 5] and 2. In 6, a different approach is taken than what we take here; in particular, the definition of the structure sheaf is different. Buium 3 has yet another approach. In that work, a differential scheme is a scheme whose structure sheaf consists of differential rings. Further, the theory of differential schemes was developed in [12, 13.

We will use the definition of a structure sheaf $\mathcal{O}$ on the differential spectrum $X$ of a differential ring $A$ as in [12, 13. It turns out that the ring of global sections of the structure sheaf does not necessarily coincide with the initial ring. Then, it is natural to ask whether the differential spectrum of the ring of global sections $\mathcal{O}(X)$ coincides with the differential spectrum of the initial differential ring $A$. Several situations in which the desired equality holds are described in [12, Proposition 10.5]. However, the most interesting case is the case of Ritt algebras, that is, differential rings containing $\mathbb{Q}$. [13, Example 7.4] shows that [12, Proposition 10.5] cannot be applied to all Ritt algebras. We resolve this issue for a wider class of differential rings - the Keigher rings [12, Definition 2.3].

The idea of the proof is the following. First, we modify the structure sheaf to the one that is both easier for us to deal with and does not lose the information about the differential spectrum. Using a simple topological fact, we show that the differential spectrum of global sections of the new sheaf coincides with that of the initial ring. In our method, the hypothesis that the ring is a Keigher ring is used essentially.

Received by the editors July 25, 2009 and, in revised form, December 5, 2010.

2000 Mathematics Subject Classification. Primary $12 \mathrm{H} 05$.

The author was partially supported by NSF grants CCF-0964875 and 0952591.

(C)2011 American Mathematical Society Reverts to public domain 28 years from publication 
The paper is organized as follows. In Section 1 we recall some basic definitions taken from [12] and [13]. In Section 2, we construct the above-mentioned auxiliary sheaf and investigate its properties. The topological result is presented in Lemma 2.1 and the main result of the section is in Theorem 2.6. In Section 3, we prove the main result (Theorem 3.2 ). Corollary 3.3 gives the answer in the case of Ritt algebras.

\section{TERMS AND NOTATION}

Throughout the text the word ring means an associative commutative ring with a unit. All homomorphisms preserve the unit. A differential ring is a ring with finitely many pairwise commuting derivations. A differential ring is called a Keigher ring if for any differential ideal $\mathfrak{a}$ its radical $\mathfrak{r}(\mathfrak{a})=\left\{x \mid x^{n} \in \mathfrak{a}\right\}$ is a differential ideal too. For any differential ring $A$, the set of all prime differential ideals is denoted by $\operatorname{Spec}^{\Delta} A$ and is called the differential spectrum of $A$. For any subset $E \subseteq A$, the set of all prime differential ideals containing $E$ is denoted by $V(E)$. Defining $V(E)$ as closed subsets, we provide the differential spectrum with the Kolchin topology. For any subset $E \subseteq A$, the smallest radical differential ideal containing $E$ will be denoted by $\{E\}$.

Denote the differential spectrum of $A$ by $X$ and let $s \in A$. Then, the set of all prime differential ideals not containing $s$ is denoted by $X_{s}$. For the set $S=\left\{s^{n}\right\}_{n=0}^{\infty}$, the localization $S^{-1} A$ is denoted by $A_{s}$. If $\mathfrak{p}$ is a prime ideal, then we denote the $\operatorname{ring} S^{-1} A$ by $A_{\mathfrak{p}}$, where $S=A \backslash \mathfrak{p}$. For any differential homomorphism $f: A \rightarrow B$, we define the corresponding map

$$
f^{*}: \operatorname{Spec}^{\Delta} B \rightarrow \operatorname{Spec}^{\Delta} A, \quad f^{*}(\mathfrak{p})=f^{-1}(\mathfrak{p}) .
$$

We will briefly recall the notion of a structure sheaf on the differential spectrum of a differential ring $A$. Consider the set of all functions

$$
\left\{f: X \rightarrow \bigcup_{\mathfrak{p} \in X} A_{\mathfrak{p}} \mid f(\mathfrak{p}) \in A_{\mathfrak{p}}\right\} .
$$

A function $f$ will be called regular at a point $\mathfrak{p}$ if there exists an open neighborhood $U$ of $\mathfrak{p}$ and $a, b \in A$, where for all $\mathfrak{q} \in U$ we have $b \notin \mathfrak{q}$, such that for all $\mathfrak{q} \in U$ it follows that $f(\mathfrak{q})=a / b$ in $A_{\mathfrak{q}}$. The set of all functions that are regular at all points of the open subset $U$ will be denoted by $\mathcal{O}(U)$. The family $\mathcal{O}(U)$ with the restriction homomorphisms forms a sheaf of differential rings [12, Section 4]. We will call this sheaf a structure sheaf.

The ring $\mathcal{O}(X)$ will be denoted by $\widehat{A}$. There is a map $\iota: A \rightarrow \widehat{A}$ such that $a \mapsto \varphi$, where $\varphi(\mathfrak{p})=a / 1$ in $A_{\mathfrak{p}}$ One can show that $\iota$ is a differential homomorphism. Further details about the structure sheaf can be found in $[12$.

\section{Auxiliary SheaF}

In this section, we construct another sheaf for which the desired problem will be solved by a straightforward calculation. The general proof given in Section 3 is based on a reduction of the initial problem to the problem solved in this section.

Let $A$ be an arbitrary differential ring. For any prime differential ideal $\mathfrak{p}$, its residue field will be denoted by $K(\mathfrak{p})$, that is, the fraction field of $A / \mathfrak{p}$. Consider 
the set of all functions

$$
\left\{f: X \rightarrow \bigcup_{\mathfrak{p} \in X} K(\mathfrak{p}) \mid f(\mathfrak{p}) \in K(\mathfrak{p})\right\} .
$$

A function $f$ will be called regular at a point $\mathfrak{p}$ if there exists an open neighborhood $U$ of $\mathfrak{p}$ and $a, b \in A$, where for all $\mathfrak{q} \in U$ we have $b \notin \mathfrak{q}$, such that for all $\mathfrak{q} \in U$ it follows that $f(\mathfrak{q})=a / b$ in $K(\mathfrak{q})$. The set of all functions that are regular at all points of $U$ will be denoted by $\mathcal{O}^{\prime}(U)$. The family of all such rings with the restriction homomorphisms forms a sheaf of differential rings on $X$.

Define a differential homomorphism $\iota^{\prime}: A \rightarrow \mathcal{O}^{\prime}(X)$ by the rule $a \mapsto \varphi$ such that $\varphi(\mathfrak{p})=a$ in $K(\mathfrak{p})$. Our proof is based on the following statement.

Lemma 2.1. Let $\varphi: X \rightarrow Y$ be a map of topological spaces and let two covers $U_{\alpha}$ and $V_{\alpha}$ of $X$ and $Y$, respectively, be given. Then, if for any pair of indices $\alpha$ and $\alpha^{\prime}$ the map $\varphi: U_{\alpha} \cap U_{\alpha^{\prime}} \rightarrow V_{\alpha} \cap V_{\alpha^{\prime}}$ is well-defined and is a homeomorphism, then $\varphi: X \rightarrow Y$ is a homeomorphism too.

Proof. Take $\alpha=\alpha^{\prime}$. Then, $\varphi$ is a local homeomorphism. We need to prove that $\varphi$ is injective. Let $x \in U_{\alpha}$ and $y \in U_{\alpha^{\prime}}$ be such that $z=f(x)=f(y)$. Then, $z \in V_{\alpha} \cap V_{\alpha^{\prime}}$. Since $\varphi$ is a homeomorphism of $U_{\alpha} \cap U_{\alpha^{\prime}}$ and $V_{\alpha} \cap V_{\alpha^{\prime}}$, the intersection $U_{\alpha} \cap U_{\alpha^{\prime}}$ is not empty and contains $w$ such that $\varphi(w)=z$. But $x$ and $w$ both belong to $U_{\alpha}$ and their images coincide. Thus, $x=w$. A similar argument shows that $y=w$.

Now, we will prove some auxiliary facts.

Lemma 2.2. Let $A$ and $B$ be differential rings, $\nu: A \rightarrow B$ be a differential homomorphism, and $B$ be differentially generated over $A$ by a single element $\eta$ such that there exists a family of elements $b_{1}, \ldots, b_{n}$ of $A$ with conditions $b_{k} \eta \in \nu(A)$ and $\left\{b_{1}, \ldots, b_{n}\right\}=A$. Then, the contraction map

$$
\nu^{*}: \operatorname{Spec}^{\Delta} B \rightarrow V(\operatorname{ker} \nu)
$$

is a homeomorphism.

Proof. First, replacing $A$ by $A / \operatorname{ker} \nu$, we may suppose that $\nu$ is injective. Let $X=\operatorname{Spec}^{\Delta} A$ and $Y=\operatorname{Spec}^{\Delta} B$. Now, we will divide the differential spectra into finitely many open subsets as follows:

$$
\begin{gathered}
X=X_{b_{1}} \cup \ldots \cup X_{b_{n}}, \\
Y=Y_{b_{1}} \cup \ldots \cup Y_{b_{n}} .
\end{gathered}
$$

We need to check the hypothesis of the previous lemma for the covers $\left\{X_{b_{i}}\right\}$ and $\left\{Y_{b_{i}}\right\}$. The proof is based on [1, Exercise 21 (i,ii) p. 46]. We replace $X_{b_{i}}$ by the differential spectrum of $A_{b_{i}}$ and $Y_{b_{i}}$ by the differential spectrum of $B_{b_{i}}$. From the mentioned exercise, it follows that the restriction of $\nu^{*}$ coincides with $\nu_{b_{i}}^{*}$ (see [1, Exercise 21 (ii) p. 47]).

In order to show the desired result, we will calculate both localizations $A_{b_{i}}$ and $B_{b_{i}}$. Let $b_{i} \eta=a_{i} \in A$. Consider

$$
B_{b_{i}}=(A\{\eta\})_{b_{i}}=A\left\{a_{i} / b_{i}, 1 / b_{i}\right\}=A\left\{1 / b_{i}\right\}=A_{b_{i}} .
$$

Therefore, the localization of $\nu: A \rightarrow B$ by $b_{i}$ induces the identity map

$$
\nu_{b_{i}}=I d: A_{b_{i}} \rightarrow B_{b_{i}}=A_{b_{i}} \text {. }
$$


So, the desired homeomorphism between $X_{b_{i}}$ and $Y_{b_{i}}$ has been obtained.

The last step of the proof is to show that $\nu^{*}$ induces a homeomorphism between $X_{b_{i}} \cap X_{b_{j}}$ and $Y_{b_{i}} \cap Y_{b_{j}}$. Having noted that $X_{b_{i}} \cap X_{b_{j}}=X_{b_{i} b_{j}}$ and $Y_{b_{i}} \cap Y_{b_{j}}=Y_{b_{i} b_{j}}$ and applying the previous argument with $b_{i} b_{j}$ instead of $b_{i}$, we obtain the result.

Using induction, we show the following.

Lemma 2.3. Let $A$ and $B$ be differential rings, $\nu: A \rightarrow B$ be a differential homomorphism, and $B$ be differentially finitely generated over $A$ such that for any generator $\eta$ there exist $b_{1}, \ldots, b_{n}$ satisfying $b_{k} \eta \in \nu(A)$ and $\left\{b_{1}, \ldots, b_{n}\right\}=A$. Then,

$$
\nu^{*}: \operatorname{Spec}^{\Delta} B \rightarrow V(\operatorname{ker} \nu)
$$

is a homeomorphism.

Proof. Let $B$ be differentially generated over $A$ by $\eta_{1}, \ldots, \eta_{n}$, and $B_{1}$ be the subring of $B$ differentially generated over $A$ by $\eta_{1}, \ldots, \eta_{n-1}$. Then, the homomorphism $\nu$ can be represented as the following composition:

$$
A \stackrel{\nu_{1}}{\longrightarrow} B_{1} \stackrel{\nu_{2}}{\longrightarrow} B .
$$

By Lemma 2.2. $V\left(\operatorname{ker} \nu_{2}\right)$ is homeomorphic to $\operatorname{Spec}^{\Delta} B$. By the inductive assumption, $\operatorname{Spec}^{\Delta} B_{1}$ and $V\left(\operatorname{ker} \nu_{1}\right)$ are homeomorphic, and, thus, their corresponding subspaces $V\left(\operatorname{ker} \nu_{2}\right)$ and $V(\operatorname{ker} \nu)$ are homeomorphic too. Consequently, $V(\operatorname{ker} \nu)$ and $\operatorname{Spec}^{\Delta} B$ are homeomorphic as well.

Lemma 2.4. Let $A_{\alpha}$ be a direct system of differential $A$-algebras and $\nu_{\alpha}: A \rightarrow A_{\alpha}$ be the corresponding homomorphisms. Suppose that for any $\alpha$ the map $\nu_{\alpha}^{*}$ is a homeomorphism. Then, $\nu^{*}$ is a homeomorphism, where $\nu: A \rightarrow \lim _{\rightarrow \alpha} A_{\alpha}$ is the direct limit of $\nu_{\alpha}$.

Proof. Denote $\lim _{\rightarrow \alpha} A_{\alpha}$ by $A^{\prime}$. We will first show that $\nu$ is bijective. Surjectivity: consider a prime differential ideal $\mathfrak{p}$ in $A$. Let $\mathfrak{p}_{\alpha}$ be the ideals in $A_{\alpha}$ corresponding to $\mathfrak{p}$, that is, $\nu_{\alpha}^{*}\left(\mathfrak{p}_{\alpha}\right)=\mathfrak{p}$. Then, $\lim _{\rightarrow \alpha} \mathfrak{p}_{\alpha}$ is a prime differential ideal in $A^{\prime}$ contracting to $\mathfrak{p}$.

Injectivity: Suppose that there exist two prime differential ideals $\mathfrak{q}$ and $\mathfrak{q}^{\prime}$ in $A^{\prime}$ contracting to $\mathfrak{p}$. Then, there exists $s \in A^{\prime}$ such that $s \in \mathfrak{q}^{\prime} \backslash \mathfrak{q}$. It follows from [1, Chapter 2, Exercise 14] that there are $\alpha$ and $s_{\alpha} \in A_{\alpha}$ such that $s_{\alpha}$ maps to $s$. Let $\mathfrak{q}_{\alpha}$ and $\mathfrak{q}_{\alpha}^{\prime}$ denote the contractions of $\mathfrak{q}$ and $\mathfrak{q}^{\prime}$ on $A_{\alpha}$, respectively. Then, we have $s_{\alpha} \in \mathfrak{q}_{\alpha}^{\prime} \backslash \mathfrak{q}_{\alpha}$. But both ideals contract to $\mathfrak{p}$ and belong to $A_{\alpha}$, a contradiction.

We will show now that $\nu$ is a homeomorphism. We just need to prove that the image of any principal open set is open. Let $s$ be an arbitrary element of $A^{\prime}$. We will demonstrate that the image of $\operatorname{Spec}^{\Delta}\left(A^{\prime}\right)_{s}$ is open in $\operatorname{Spec}^{\Delta} A$. Indeed, for some $\alpha$, there is $s_{\alpha} \in A_{\alpha}$ such that its image coincides with $s$. Then,

$$
\operatorname{Spec}^{\Delta}\left(A_{\alpha}\right)_{s_{\alpha}}=\operatorname{Spec}^{\Delta}\left(A^{\prime}\right)_{s} .
$$

But, by the data, the image of $\operatorname{Spec}^{\Delta}\left(A_{\alpha}\right)_{s_{\alpha}}$ is open in $\operatorname{Spec}^{\Delta} A$, which finishes the proof.

Lemma 2.5. For every $\eta \in \mathcal{O}^{\prime}(X)$, there exist $b_{1}, \ldots, b_{m} \in A$ such that $b_{i} \eta \in \iota^{\prime}(A)$ and $\left\{b_{1}, \ldots, b_{m}\right\}=A$.

Proof. Without the loss of generality, we may suppose that $U_{1} \cup \ldots \cup U_{m}=X$ such that $U_{i}=X_{b_{i}}$ and, for any $\mathfrak{p} \in U_{i}$ we have $\eta(\mathfrak{p})=a_{i} / b_{i}$ in $K(\mathfrak{p})$. We will replace $a_{i}$ and $b_{i}$ by $a_{i} b_{i}$ and $b_{i}^{2}$, respectively. Then, for any $\mathfrak{p} \notin U_{i}$, we have $a_{i}=0$ in $K(\mathfrak{p})$. 
Then, since all $U_{i}$ cover $X$, it follows that $\left\{b_{1}, \ldots, b_{m}\right\}=A$. Let us show that, for every $\mathfrak{p}, b_{i} \eta(\mathfrak{p})=a_{i}$ in $K(\mathfrak{p})$ holds. Indeed, if $\mathfrak{p} \in U_{i}$, then

$$
b_{i} \eta(\mathfrak{p})=b_{i}\left(a_{i} / b_{i}\right)=a_{i}
$$

in $K(\mathfrak{p})$. If $\mathfrak{p} \in U_{j} \backslash U_{i}$, then

$$
b_{i} \eta(\mathfrak{p})=b_{i}\left(a_{j} / b_{j}\right)=0
$$

in $K(\mathfrak{p})$, but $a_{i}=0$ in $K(\mathfrak{p})$ too. Consequently, $b_{i} \eta=\iota^{\prime}\left(a_{i}\right)$.

Theorem 2.6. Let $D$ be a differential subring of $\mathcal{O}^{\prime}(X)$ containing the image of $A$ and $\iota^{\prime}: A \rightarrow D$ be the corresponding homomorphism. In this case,

$$
\iota^{\prime *}: \operatorname{Spec}^{\Delta} D \rightarrow \operatorname{Spec}^{\Delta} A
$$

is a homeomorphism.

Proof. It follows from Lemma 2.5 that for any differentially finitely generated over $A$ subalgebra in $\mathcal{O}^{\prime}(X)$ the hypotheses of Lemma 2.3 hold. From the definition of $\iota^{\prime}$, it follows that ker $\iota^{\prime}$ belongs to the intersection of all prime differential ideals of $A$. Consequently, for any differentially finitely generated algebra $D$ the statement is proven. But any differential algebra can be presented as a direct limit of its differentially finitely generated subalgebras. Then, it follows from Lemma 2.4 that the statement holds for any subalgebra $D$.

\section{MAIN RESUlt}

In this section, we reduce the main result to Theorem 2.6. We have two sheaves on the topological space $X$, namely $\mathcal{O}$ and $\mathcal{O}^{\prime}$. Consider the homomorphism of sheaves

$$
\varphi(U): \mathcal{O}(U) \rightarrow \mathcal{O}^{\prime}(U), \quad \varphi(f)(\mathfrak{p})=f(\mathfrak{p}) \quad \bmod \mathfrak{p} .
$$

From now on we suppose that the given ring $A$ is a Keigher ring.

Proposition 3.1. With the above conventions, $\operatorname{ker} \varphi(X)$ coincides with the nilradical of $\mathcal{O}(X)$.

Proof. By definition, $\operatorname{ker} \varphi(X)$ coincides with

$$
\left\{f \in \mathcal{O}(X) \mid f(\mathfrak{p}) \in \mathfrak{p} A_{\mathfrak{p}}\right\} .
$$

Let us show that the nilradical is contained in the kernel. Let $f$ be a nilpotent element. Then, for any $\mathfrak{p}$ we have $f^{n}(\mathfrak{p})=0$. So, $f^{n}(\mathfrak{p}) \in \mathfrak{p} A_{\mathfrak{p}}$. Consequently, $f(\mathfrak{p}) \in \mathfrak{p} A_{\mathfrak{p}}$.

Conversely, let $f \in \operatorname{ker} \varphi(X)$. Then, there exists a cover $U_{1} \cup \ldots \cup U_{n}=X$ such that for every $i$ we have $f(\mathfrak{p})=a_{i} / b_{i}$ in $A_{\mathfrak{p}}$, whenever $\mathfrak{p} \in U_{i}$. Without loss of generality we may suppose that $U_{i}=X_{b_{i}}$. Fix some $i$. Then,

$$
f(\mathfrak{p})=a_{i} / b_{i} \in \mathfrak{p} A_{\mathfrak{p}},
$$

for all $\mathfrak{p}$ not containing $b_{i}$. Then, $a_{i} \in \mathfrak{p}$ for all $\mathfrak{p}$ not containing $b_{i}$. Therefore, $a_{i}$ belongs to the intersection of all prime ideals in $A_{b_{i}}$. Since $A$ is a Keigher ring $\left(A_{b_{i}}\right.$ too), $a_{i}$ belongs to the nilradical of $A_{b_{i}}$ [12, Corollary 2.9]. Hence, for some $k_{i}$ we have $a_{i}^{k_{i}}=0$ in $A_{b_{i}}$. Therefore, for some $m_{i}$, it follows that

$$
b_{i}^{m_{i}} a_{i}^{k_{i}}=0
$$


in $A$, and so we have that $\left(a_{i} b_{i}\right)^{n_{i}}=0$ for some $n_{i}$. Let $n=\max _{i} n_{i}$. Then, for every $i$ we have $\left(a_{i} b_{i}\right)^{n}=0$ in $A$. Consequently, for every $i$ and every $\mathfrak{p} \in U_{i}$ the following holds:

$$
f^{n}(\mathfrak{p})=\left(\frac{a_{i}}{b_{i}}\right)^{n}=\frac{\left(a_{i} b_{i}\right)^{n}}{b_{i}^{2 n}}=0 \text { in } A_{\mathfrak{p}}
$$

Theorem 3.2. Let $D$ be a differential subring of $\mathcal{O}(X)$ containing the image of $A$ and $\iota: A \rightarrow D$ be the corresponding homomorphism. Then,

$$
\iota^{*}: \operatorname{Spec}^{\Delta} D \rightarrow \operatorname{Spec}^{\Delta} A
$$

is a homeomorphism.

Proof. Consider the homomorphism $\varphi(X): D \rightarrow \mathcal{O}^{\prime}(X)$. It follows from Proposition 3.1 that the kernel of $\varphi(X)$ coincides with the nilradical of $D$. The quotient of $D$ by its nilradical will be denoted by $D^{\prime}$. It is clear that $\operatorname{Spec}^{\Delta} D$ can be identified with $\operatorname{Spec}^{\Delta} D^{\prime}$. We have the following sequence of homomorphisms:

$$
A \rightarrow D^{\prime} \rightarrow \mathcal{O}^{\prime}(X)
$$

and the composition coincides with $\iota^{\prime}$. It follows from Theorem 2.6 that $\operatorname{Spec}^{\Delta} D^{\prime}$ $\rightarrow \operatorname{Spec}^{\Delta} A$ is a homeomorphism. Remembering that $\mathrm{Spec}^{\Delta} D$ is homeomorphic to $\operatorname{Spec}^{\Delta} D^{\prime}$, we obtain the desired result.

Corollary 3.3. Let $A$ be a Ritt algebra and $\iota: A \rightarrow \widehat{A}$ be the canonical homomorphism into the ring of global sections. Then,

$$
\iota^{*}: \operatorname{Spec}^{\Delta} \widehat{A} \rightarrow \operatorname{Spec}^{\Delta} A
$$

is a homeomorphism.

\section{ACKNOWLEDGMENT}

The author thanks the referee for very helpful suggestions.

\section{REFERENCES}

1. M. F. Atiyah, I. G. Macdonald. Introduction to commutative algebra. Addison-Wesley, 1969. MR0242802(39:4129)

2. A. Buium. Ritt schemes and torsion theory. Pacific Journal of Mathematics 98 (1982), pp. 281-293. MR650009 (84j:13028)

3. A. Buium. Differential function fields and moduli of algebraic varieties. Lecture Notes in Mathematics 1226, Springer-Verlag, Berlin-New York, 1986. MR874111 (88e:14010)

4. G. Carrà Ferro. Sullo spettro differenziale di un anello differenziale. Le Mathematiche (Catania) 33 (1978), pp. 1-17. MR612425 (82h:13018)

5. G. Carrà Ferro. The ring of global sections of the structure sheaf on the differential spectrum. Rev. Roumaine Math. Pures Appl. 30 (1985), pp. 809-814. MR826241 (87j:13036)

6. G. Carrà Ferro. Kolchin schemes. Journal Pure and Applied Mathematics 94 (1972), pp. 891-954.

7. W. Keigher. Adjunctions and comonads in differential algebra, Pacific Journal of Mathematics 59 (1975), pp. 99-112. MR0392957 (52:13770)

8. W. Keigher. Prime differential ideals in differential rings. Contribution to Algebra: A collection of papers dedicated to Ellis Kolchin (Bass, Cassidy, Kovacic, eds.), Academic Press, New York, 1977, pp. 239-249. MR0485806 (58:5610)

9. W. Keigher. On the structure presheaf of a differential ring. Journal of Pure and Applied Algebra 27 (1983), pp. 163-172. MR687748 (84g:13040)

10. W. Keigher. On the quasi-affine scheme of a differential ring. Advances in Mathematics 42(2) (1981), pp. 143-153. MR641423 (83c:12023) 
11. W. Keigher. Differential schemes and premodels of differential fields. Journal of Algebra 79 (1982), pp. 37-50. MR679968 (84f:13022)

12. J. J. Kovacic. Differential Schemes, Differential Algebra and Related Topics, Newark, NJ, 2000, pp. 71-94. MR1921695 (2003i:12010)

13. J. J. Kovacic. Global sections of diffspec, Journal of Pure and Applied Algebra 171 (2002), pp. 265-288. MR.1904483 (2003c:12008)

Department of Mechanics and Mathematics, Moscow State University, Moscow, Russia 119991

E-mail address: trushindima@yandex.ru 